\title{
YOUR DESIGN PROBABLY NEEDS MORE VDU'S
}

\author{
John O'Hara and William Brown \\ Brookhaven National Laboratory \\ Upton, New York
}

\author{
Paul Lewis and J. Persensky \\ U.S. Nuclear Regulatory Commission \\ Washington, D.C.
}

\begin{abstract}
The most frequent complaint of operators in modern computer-based control rooms is that there just are not enough video display units (VDUs). In this paper we examine the basis for this concern and try to understand the technical and historical reasons for this complaint, and its implications for the design of complex human-machine systems, including the number of VDUs in the control room. The overall aim of our work is to develop human factors guidance for the review of computer-based and modernized control rooms in nuclear power plants. As part of these efforts we have conducted literature reviews and studies using both simulators and actual systems in a broad range of industries, including process control, aerospace, medical, and others. Our findings reflect the general complaint of operators across all these industries: there just are not enough VDUs in the control room. We conclude that there are three primary reasons for this complaint. First, as part of a workload management strategy, operators frequently avoid interface management tasks and do not access all the information available, preferring instead to use a fixed set of familiar displays that provide much (but not all) of the information needed. Performance thereby becomes data limited and operators complain that they do not have a sufficient number of VDUs to set up in the early phases of a high-workload period so they can get all the information they need. Second, display designs are typically not designed with operator tasks in mind. The most common method of representing information is by functions and systems. Since tasks typically cut across many systems, operators need many displays. Thus, to make task performance easier operators need additional VDUs. Finally, there is a differing "concept of operations" between designers and operators. Modern computer-based control rooms are designed with vast amounts of data, available through hundreds of displays, viewed by the operator through a limited number of display devices. Designers expect that operators will use the flexibility of the computer-based interfaces to configure them in such a way that they are ideally tailored to the unique demands of the current situation. However, operators usually do not do that and instead configure the interfaces in a spatially dedicated way. Thus, while the number of VDUs may seem reasonable to the designer, it is not to the operator who is attempting to minimize the interface management aspects of workload. The implications of these findings for design are discussed in terms of the need for a method for determining the number of displays, task-relevant displays, data-dense displays, and enhanced interface management design and training.
\end{abstract}

\section{INTRODUCTION}

Control rooms of the past were typically very large workspaces with spatially dedicated human-system interfaces (HSIs); i.e., alarms, displays, and controls. In this context, spatial dedication means that individual HSI components are always in the same place and visible to operators in their vicinity. Operators "walked the boards" to monitor information and perform plant control operations from a standing position. Operators integrated and interpreted information based on their training, experience, and crew communication.

By contrast, computer-based control rooms provide many HSIs through video display units (VDUs). These HSI usually lack spatial dedication and exist in a virtual rather than physical workspace. The workplace is considerably more compact and includes workstation-like consoles for seated operators. The underlying digital instrumentation and control systems are capable of providing much more data to operators. The information systems may have thousands of display pages. Operators view a small amount of this information at any one time through their VDUs. The characteristic of limited viewing area sometimes has been referred to as the "keyhole effect" (Woods, 1990). Sometimes, additional display area is provided via large "wall panel" displays that can be viewed by the entire crew.

We have been studying control room modernization and the effects of advanced technology on crew performance. As part of these efforts we have conducted literature reviews and studies in a broad range of industries, including process control, aerospace, medical, and others. The results have been used to develop human factors guidance for the review of computer-based and modernized control rooms in nuclear power plants (O'Hara, Brown, et al., 2000).

One interesting and consistent finding across industries, is that operators comment that there are just not enough VDUs in their computer-based control rooms. In this paper we will examine the basis for this concern and will discuss its implications for how complex humanmachine systems are designed. 


\section{METHODOLOGY}

To explore this hypothesis, we used the following sources of information (1) literature analysis, (2) interviews with subject matter experts from many industrial domains, (3) walkdowns of scenarios in seven process control facilities, and (4) two simulator studies focused on these and related issues (Roth and O'Hara, 1998; O'Hara, et al., 2000). A complete discussion of these information sources and the methods used in this study is contained in O'Hara, Brown, Stubler, and Lewis (in press).

\section{FINDINGS}

\section{Advantages of Limiting the Number of VDUs}

There are advantages to limiting the number of VDUs in a control room from a design perspective. Fewer VDUs (or more properly less display area) means smaller control rooms, more simplicity in that there are fewer HSIs to integrate, less cost for equipment, and a lower maintenance burden. In operational environments with significant space and power limitations, such as air and space craft, designing HSIs with fewer VDUs may be a logistical necessity. However, except in these specialized situations, the advantages of fewer VDUs may not be justified when considering the cost to operating crew performance.

\section{Workload Management}

The primary tasks of operators in complex systems involve generic cognitive activities, i.e., situation assessment, monitoring and detection, response planning, and response implementation. To access the controls and displays necessary to perform primary tasks, operators are required to perform interface management (IM) tasks, i.e., actions performed by the operator to interact with the HSIs such as display navigation and window manipulation.

Based on the dual-task literature, one might predict that there is a cognitive cost for accessing information (Wickens and Carswell, 1995; Wickens and Seidler,1997). IM tasks are potentially distracting secondary tasks that impose demands on memory and may interfere with primary task performance. Thus time spent on IM tasks is time that is not available for primary tasks.

However, much of the dual-task literature is based on laboratory research examining divided attention where the two tasks are relatively independent, i.e., performance of one is not dependent on the other. This is not the case in modern control rooms. IM tasks must be performed in order to retrieve the information relevant to the operator's ongoing activities.

We investigated the effects of IM on primary task performance in multi-task environments such as control centers of complex systems by first identifying models of how IM tasks could affect primary task performance. Norman and Bobrow (1975) indicated that to adequately perform tasks, two conditions must be met: (1) cognitive resources (such as attention, reasoning, and memory) must be available, and (2) adequate information about system performance must be available. This relationship has been referred to as a performance-resource function. Multipleresource theory suggests the amount of cognitive resources available to support task performance is finite (Wickens, 1984). When the resources demanded exceed those supplied, performance declines. System information is needed as well. No matter how many cognitive resources are available, if the information needed to effectively monitor and control the system is too limited, task performance degrades.

In computer-based control rooms, primary tasks and IM tasks rely on many of the same cognitive resources and use many of the same HSIs (e.g., a mouse may be use to both start a pump and retrieve displays). Thus they compete for resources and a dual-task situation arises; resources can be devoted to one or the other task, or they can be divided between them. Based on these considerations and the dependent nature of the two classes of tasks, three hypothetical dual-task performance models under cognitively demanding situations were defined:

- Resource-limited model - IM tasks draw resources away from primary task performance, and primary task performance becomes resource limited and declines.

- Data-limited model - Primary tasks consume most of the cognitive resources leaving little for IM performance. Since the primary tasks are dependent on IM tasks, primary task performance becomes data limited and declines when IM tasks are not performed.

- Divided-attention model - Cognitive resources are shared between the primary and secondary tasks, and primary task performance can be both resource and data limited.

These models were then evaluated to determine whether they described actual effects involving IM tasks in complex systems. The information analyzed came from the sources identified in the Methodology section above. Interestingly, we found that very few studies have been performed specifically examining this issue in complex task domains. We did however find evidence for effects of IM on performance across a variety of data sources. Support for resource-limiting and divided-attention effects were found, although the available data could not distinguish between them. IM tasks can draw cognitive resources away from primary tasks. Operators frequently comment that IM tasks are cognitively demanding and require them to have more knowledge and skill related to the use of the HSI when compared with older analog HSIs. They would not have to perform these demanding tasks as often if they had more VDUs.

Support was also found for the data-limited effect. As part of a workload management strategy, operators frequently avoid IM tasks and do not access all the 
information available, preferring instead to use a fixed set of familiar displays that provide much (but not all) of the information needed. Performance thereby becomes data limited and operators complain that they do not have a sufficient number of VDUs to set up in the early phases of a high-workload period.

In summary, interface management tasks may create barriers between operators and important information. During periods of high workload, such as major disturbances, operators, in certain circumstances, may decide to not access additional information because the retrieval effort may detract from the operators' primary task of analyzing the situation. Also, selecting new displays may disrupt ongoing tasks or may interfere with current information being used.

\section{Display Designs are Not Well Suited to Operator Tasks}

In computer-based control rooms, the most common method of representing information is by functions and systems. Organizing displays in such a manner was effective in older control rooms because the spatial dedication allowed operators to scan the boards. However, limiting display organization in this way may not be effective for computer-based control rooms (Heslinga and Herbert, 1995). For routine operations that occupy the vast majority of an operator's time, accessing the information they need can be a difficult, effortful task. The reason for this is that for more routine tasks, operators' information needs are not centered along the system hierarchy. Since tasks typically cut across many systems operators need to access many displays to retrieve task information. When the viewing area is limited, they are required to perform many IM tasks. In particularly bad situations, operators may be forced to make repetitive transitions among displays, an action that is sometimes referred to as display thrashing (Henderson and Card, 1987). Operators need additional VDUs so they can have access to the information they need in parallel.

\section{Differing Concept of Operations}

Operators often do not use HSIs in ways that designers expect and they adopt numerous strategies to create workarounds and aids to correct for limitations in designs (Cook, Woods, and Howie, 1990; Woods, Johannesen, Cook, and Sarter, 1994; Vicente et al., 1997). If the datalimited model accurately characterizes an effect of interface management, then an interesting paradox is created. As noted above, control rooms are designed with vast amounts of data, available through hundreds and sometimes thousands of displays, viewed by the operator through a limited number of display devices. Designers may expect that operators will use the flexibility of the computer-based interfaces to configure the HSI in such a way that it is ideally tailored to the unique demands of the current situation. However, as we have seen operators frequently prefer not to do that. Being reluctant to perform such tasks, they instead configure their HSI as a spatially dedicated one. This behavior is a part of their workload management strategy. Thus, while the number of VDUs may have seemed reasonable to the designer when considering the flexibility and interface management and configuration resources provided in the control room, it is not to the operator who is attempting to minimize this aspect of workload in order to maximize the time available to perform primary tasks. The operator would rather have more VDUs so that their initial "set-up" can display all the information they will need.

\section{DISCUSSION AND DESIGN IMPLICATIONS}

\section{Methods for Determining the Number of Displays}

Very often one of the first details pinned down is the overall control room configuration and workstation layout, e.g., configurations of hardware, such as alarms, monitors, keyboards, and switches. The designers determine how many monitors will be available before they know what information will be presented to the operator or how it will be presented. This is often determined before input from operational crews is sought. There is only a loose coupling between information needs, information presentation, and display area needed to support operations.

Determining the appropriate amount of display area should include determining the information that will be needed at one time by the operators, the arrangement of information within display pages, the arrangement of pages within the display network, and the means used to access the information. It should also take into account the coordination of activities across crew members. For example, some displays may be shared by multiple operators at a workstation, which may reduce the total number needed. Alternatively, additional display devices may be needed to present group-view displays to support communication and coordination among personnel. When HSI design requirements are developed, these factors should be evaluated to determine the number of VDUs that will reduce the overall cost associated with accessing information during peak workload conditions. Designs should reflect the fact that IM tasks may not always be performed.

\section{Task-Relevant Displays}

Displays that provide better support for task performance will help minimize the number of VDUs that are necessary for task performance by bringing more taskrelevant information together in single displays. This will help minimize the need for operators to access multiple displays and place them on individual VDUs or on individual windows within a VDU. An example of a task-oriented display is a display that is structured according to operational or emergency procedures an operator must follow (O'Hara, Higgins, et al., 2000). 
This discussion should not be interpreted to suggest the other forms of representation (e.g. function- and system-based) are not needed. They are needed for overall monitoring of system statue, fault diagnosis, and handling situations that are unplanned and unanticipated by designers. The point is that for the types of activities that take up most of the operator's time, the absence of taskoriented displays impairs crew performance.

\section{Data-Dense Displays}

The number of VDUs in the control room can be reduced if more information is placed on individual display pages. However, dense displays are usually considered undesirable. Thus a tradeoff is created between distributing information over many less-dense displays that require a lot of navigation and packing displays with data potentially resulting in a crowded appearance but requiring less navigation.

Techniques that support mental integration of displayed items, such as placing task-related items close together, grouping task-related items, and integrating alphanumerics and graphics into visual objects, may enhance performance while actually increasing display density. Newer display forms such as integral and configural displays (Bennett, et al., 1997) may greatly increase display density while reducing information access costs and improving user performance.

It has been found that displays which initially appear crowded to operators can become well liked and effective in supporting performance as operators gain experience with them (Roth, et al., 1998).

\section{Enhanced Interface Management Design and Training}

In part, the demand for VDUs goes up as the resources available for interface management performance goes down. Thus, designing interface management to be less effortful and demanding can minimize the need for additional VDUs and enable operators to better concentrate on their primary tasks. Some of the ways this can be accomplished include:

- Organizing display networks so operators can easily understand them and so that information can be readily located and retrieved.

- Providing enhanced navigation functions such as landmarks, embedded links, transition pointers, and history functions.

- Automating aspects of interface management, such as display retrieval.

- Improving training in interface management strategies.

\section{CONCLUSION}

While there may be advantages to limiting the number of VDUs in a control room, they may be offset by their effects on operators. We found operators' concern about limited display area to be a very common issue and one that transcended domain application. The trend to pack more and more information and functionality into computer-based systems, while at the same time reducing the ability to display information simultaneously makes operational tasks difficult. In fact, in hybrid control rooms where operators have a choice to use "old fashion," spatially dedicated HSIs or computer-based HSIs operators will frequently opt for the spatially dedicated HSIs when in difficult situations. If your control room evolved the way that most of the control rooms we studied did (where numbers of displays were determined prior to knowing how information will be structured and used), if it utilizes the types of display formats that we found commonly employed (not task-oriented), and if the design is based on the premise that operators can perform interface management tasks to retrieve the most task appropriate information and configure it whatever way they want to, then your design probably needs additional VDUs.

By increasing the number of VDUs, operators can display more information at any one time, thus reducing the demand to remember information from one display to another and reducing the need for interface management tasks to retrieve and configure information. With additional VDUs operators can also have the opportunity to use some VDUs as spatially dedicated displays so they can put overview displays or specific information, such as parameter trends, that may be important to the current ongoing task.

Further, by using many of the other design techniques discussed above, such as building more task-oriented/datadense displays and providing operators with better training on IM strategies, the need for additional VDUs can be reduced. Finding the right balance between the number of VDUs and better information system design can lead to a more effective and usable control room design.

\section{ACKNOWLEDGMENTS}

This research is being sponsored by the NRC. The views presented in this paper represent those of the authors alone, and not necessarily those of the NRC.

\section{REFERENCES}

Bennett, K., Nagy, A., \& Flach, J. (1997). Visual displays. In G. Salvendy (Ed.), Handbook of human factors and ergonomics (Second Edition). New York, NY: John Wiley and Sons.

Cook, R., Woods, D., \& Howie, M. (1990). The natural history of introducing new information technology into a high risk environment. In Proceedings of the Human Factors and Ergonomics Society - 34th Annual Meeting. Santa Monica, CA: Human Factors and Ergonomics Society.

Henderson, A. \& Card, S. (1987). A multiple, virtualworkspace interface to support user task switching. In Proceedings of the CHI'87 Human Factors in Computing Systems Conference. New York, NY: 
Association for Computing Machinery.

Heslinga, G. \& Herbert, M. (1995). Experiences with advanced systems for human-machine interaction. In Proceedings of the 6th IFAC/IFIP/IFORS/IEA Symposium on Analysis, Design and Evaluation of Man-Machine Systems. Cambridge, MA: Massachusetts Institute of Technology.

Norman, D. \& Bobrow, D. (1975). On data-limited and resource-limited processes. Cognitive Psychology, 7, 44-64.

O'Hara, J., Brown, W., Stubler, W., \& Lewis, P. (in press). The Effects of Interface Management Tasks on Crew Performance and Safety in Complex, ComputerBased Systems. (Draft NUREG/CR). Washington, D.C.: U.S. Nuclear Regulatory Commission.

O'Hara, J., Brown, W., Hallbert, B., Skråning, G., Wachtel. J., \& Persensky, J. (2000). The effects of alarm display, processing, and availability on crew performance (NUREG/CR-6691). Washington, D.C.: U.S. Nuclear Regulatory Commission.

O'Hara, J., Brown, W., Higgins, J., Stubler, W., Wachtel. J., \& Persensky, J. (2000). Human factors guidance for control room evaluation. In Proceedings of the IEA2000/HFES200 Congress (Vol. 3) (pp. 519-522). Santa Monica, CA: Human Factors and Ergonomics Society.

O'Hara, J., Higgins, J., Stubler, W., \& Kramer, J. (2000). Computer-based procedure systems: Technical basis and human factors review guidance (NUREG/CR6634). Washington, D.C.: U.S. Nuclear Regulatory Commission.
Roth, E. \& O'Hara, J. (1998). Integrating digital and conventional human system interface technology: Lessons learned from a control room modernization program. (BNL Report J6012-3-4-7/98). Upton, New York: Brookhaven National Laboratory.

Roth, E., Lin, L., Thomas, S., Kerch, S., Kenney S., \& Sugibayashi, N. (1998). Supporting situation awareness of individual displays and teams using group view displays. In In Proceedings of the Human Factors and Ergonomics Society - 42nd Annual Meeting (pp. 244-248). Santa Monica, CA: Human Factors and Ergonomics Society.

Vicente, K., Mumaw, R. \& Roth, E. (1997). Cognitive functioning of control room operators. Toronto, Canada: University of Toronto.

Wickens, C. \& Carswell, C. (1997). Information processing. In G. Salvendy (Ed.), Handbook of human factors and ergonomics (Second Edition). New York, NY: Wiley-Interscience.

Wickens, C. and Seidler, K. (1997). Information access in a dual-task context: Testing a model of optimal strategy selection. Journal of Experimental Psychology: Applied, 3, 196-215.

Woods, D., Johannesen, L., Cook, R., and Sarter, N. (1994). Behind human error: Cognitive systems, computers, and hindsight (CSERIAC SOAR 94-01). Wright Patterson Air Force Base, Ohio: Crew Systems Ergonomics Information Analysis Center.

Woods, D., Roth, E., Stubler, W., and Mumaw, R. (1990). Navigating through large display networks in dynamic control applications. In Proceedings of the Human Factors Society - 34th Annual Meeting. Santa Monica, CA: Human Factors and Ergonomics Society. 